\title{
MODULATION OF AUTONOMIC NERVOUS SYSTEM ASSESSED THROUGH HEART RATE VARIABILITY BY INTEGRATED AMRITA MEDITATION TECHNIQUE IN TYPE 2 DIABETIC SUBJECTS - A PILOT STUDY
}

\author{
SARIKA KS ${ }^{1}$, VANDANA BALAKRISHNAN ${ }^{1 *}$, HARISH KUMAR ${ }^{2}$, ANAND KUMAR ${ }^{3}$, KR SUNDARAM ${ }^{4}$ \\ ${ }^{1}$ Department of Physiology, Amrita Institute of Medical Sciences, Amrita Lane, Cochin, Kerala, India. ${ }^{2}$ Department of Endocrinology, Amrita \\ Institute of Medical Sciences, Amrita Lane, Cochin, Kerala, India. ${ }^{3}$ Department of Neurology, Amrita Institute of Medical Sciences, Amrita \\ Lane, Cochin, Kerala, India. ${ }^{4}$ Department of Biostatistics, Amrita Institute of Medical Sciences, Amrita Lane, Cochin, Kerala, India. \\ Email: vandanabalakrishnan@aims.amrita.edu \\ Received: 13 June 2020, Revised and Accepted: 23 July 2020
}

ABSTRACT

Objective: This study aims in understanding the effects of Integrated Amrita Meditation (IAM), a type of mindfulness meditation, on the autonomic balance of type 2 diabetic patients through assessment of heart rate variability (HRV).

Methods: After the initial screening of 30 type 2 diabetic subjects, 10 type 2 diabetic subjects between the age group of 30 and 65 years were randomized into two groups, diabetic test $(n=5)$ and diabetic control group $(n=5)$. Diabetic test group practiced IAM technique under the guidance of a trained practitioner. Both the groups continued the same dietary pattern and medications during the 6-month study period. HRV was taken for all subjects at baseline and after 6 months. In our study, we have focused on the power spectral analysis of HRV which include normalized units of high frequency (nHF), low frequency (nLF), and low frequency-high frequency ratio (LF/HF ratio).

Results: Mean percentage change in nHF, nLF, and LFHF ratio showed significant changes in between-group comparison ( $<<0.05)$. Normalized units of HF increased ( $\mathrm{p}=0.049)$ while LF $(\mathrm{p}=0.036)$ and LFHF ratio $(\mathrm{p}=0.024)$ decreased significantly within test group after 6 months of IAM practice suggesting the potential of IAM in improving the parasympathetic tone, thereby tuning the mind and body to calm down during stress.

Conclusion: Our study has shown demonstrable improvement in autonomic function which reflects reduced stress after the practice of IAM in diabetic patients.

Keywords: Autonomic function, Diabetes, Heart rate variability, Integrated Amrita Meditation, Meditation

(c) 2020 The Authors. Published by Innovare Academic Sciences Pvt Ltd. This is an open access article under the CC BY license (http://creativecommons. org/licenses/by/4. 0/) DOI: http://dx.doi.org/10.22159/ajpcr.2020.v13i10.38745

\section{INTRODUCTION}

The past two decades have witnessed an increase in psychological interventions through mind-body medicine in clinical practices to impart effective treatment regimens in various stress-related diseases such as diabetes, hypertension, depression, and anxiety-related disorders [1]. Unlike healthy individuals, in the patient population, especially diabetic subjects, the distress and agony of the affliction and the related worsening of disease are common and continue as a vicious circle unless their stress component is managed effectively. Hence, a better understanding of the effect of meditation on the autonomic nervous system (ANS) of an individual is crucial as the latter plays a pivotal part in regulating the emotional component within the human body. A measure of beat-to-beat variability in heart rate that is mediated by the autonomic nervous systems is referred to as heart rate variability (HRV) [2]. HRV signifies a person's capacity to balance their emotions [3] and hence can be used as a tool to portray one's emotional resilience [4].

Hon and Lee in 1965 were the first to demonstrate the clinical application of HRV. They showed that in fetal distress, disturbances in HRV were observed before changes in the heartbeat itself [5]. Later in the 1970s, HRV changes in diabetic subjects were used to predict autonomic neuropathy even before the onset of symptoms [6]. Several further studies on diabetic subjects confirmed the reduction in HRV in diabetics as compared to healthy controls. HRV is found to be lowered in diabetic patients than healthy individuals [7].

In the past two decades, there has been a rapid increase in the number of people diagnosed with diabetes worldwide $[8,9]$. Conspicuous changes in human environs, habits, and lifestyle accompanied by globalization have resulted in mounting rates of both obesity and diabetes [10]. Since Type 2 diabetes mellitus (T2DM) is a psychosomatic illness, treating the somatic and symptomatic aspects of the disease alone will not help in curing the patient. The psychiatric aspect of the disease, especially stress, must be addressed. Focusing on effective stress-relieving strategies would be of paramount importance for attaining a healthy living for diabetic patients as such interventions can help delay the progression toward severe complications by shifting the autonomic balance toward parasympathetic activity and thereby reducing the stress-induced hyperglycemia in diabetic subjects. International Diabetes Federation recommends evidence-based care as they are cost effective and can be made available to all people with diabetes [11]. Relaxation exercises such as yoga and meditation can be easily learned and practiced as it is safe, non-invasive and demanding very little in terms of equipment or professional training $[12,13]$.

Integrated Amrita Meditation ${ }^{\circledast}$ (IAM) technique, a trademarked technique designed by Mata Amritanandamayi Math (MAM), is a form of meditation whose origin is fundamental to Indian Tantric practices. It encompasses yogic postures, breathing exercise, and meditation, all three components which can be practiced easily within $23 \mathrm{~min}$. This again is conducive for the modern fast-paced life [14]. Through a 5-year study of IAM on normal healthy individuals [15], it was shown that the technique succeeded in bringing significant changes in one's attitude toward stress. Thus, understanding the potential of IAM technique in stress management, we planned to conduct this pilot study to determine the effect of IAM on the autonomic nervous system of diabetic subjects which would help us to understand the impact of meditation more deeply. 
The objective of our study was to determine the modulation of autonomic nervous system through HRV in Type 2 diabetic subjects and to compare the changes with a control group. Furthermore, this pilot study was aimed to sort out any practical problems in collecting the data on HRV and those related to the conduct of IAM meditation by the participants. This is the first study of IAM technique on patients.

\section{METHODS}

\section{Subjects}

Ten type 2 diabetic subjects between the age group of 30 and 65 years with diabetes of 1-10 years duration and whose HbA1C level ranged between 7 and $10 \%$ and who volunteered to participate in the study were recruited from Endocrinology Outpatient Department of Amrita Institute of Medical Sciences.

\section{Ethical aspects}

The study protocol conforms to ethical guidelines of the "World Medical Association Declaration of Helsinki Ethical Principles for Medical Research Involving Human Subjects" adopted by the $18^{\text {th }}$ WMA General Assembly, Helsinki, Finland, June 1964, as revised in Tokyo, 2004. The protocol received Institutional Human Research Ethical Committee and Scientific Advisory Committee clearance before the commencement and subjects signed informed consent forms before participation in the study.

The study was conducted from December 2018 to June 2019. The recruited patients had not previously undergone any specialized relaxation training and volunteered to participate in the study. Patients diagnosed with advanced diabetic complications - ongoing treatment for retinopathy/renal impairment/symptomatic or unstable heart disease/uncontrolled BP were excluded from the study.

\section{Study design}

The subjects were randomly assigned to two groups. Randomization of the study participants to both the groups was done through computergenerated sequence. Group 1 consisting of Type 2 diabetic patients receiving standard medical care and undergoing IAM technique (diabetic test group/IAM group) and Group 2 consisting of Type 2 diabetic patients receiving standard medical care alone and not undergoing any relaxation exercises (diabetic control group). IAM technique is taught by teachers who are well versed with the technique and approved as teachers by the MAM. Both the groups continued with the same dietary pattern and there was no change in medication during the study period. The study duration was for 6 months.

\section{Materials and methods of HRV data collection}

Before starting the meditation, HRV was taken in the test group subjects. All patients' data collection was done at the neurology electroencephalogram (EEG) laboratory of Amrita Institute of Medical Sciences. For the test group subjects, the data were collected on the day before the course. Since the human cardiac function is regulated differently by ANS in a day, the experiments were conducted during the same period ( $3 \mathrm{pm}-6 \mathrm{pm})$ to ensure similar states of ANS for all subjects. Subjects reported to study after refraining from food for $2 \mathrm{~h}$ and stoppage/minimizing the consumption of tobacco, alcohol, and caffeine products at least a day before the study was recommended. The commercial device used for collecting data which was Xltek Trex HD (Natus Medical Incorporated, USA). After explaining the procedures, the patient was connected with limb leads of ECG, and the parameters of HRV were obtained in supine position after 10 min of rest. The recorded data of 30 min duration were converted to European Data Format (EDF) by acquisition software and then processed using code written in MATLAB (a computer programming language). First and last $30 \mathrm{~s}$ periods of supine rest were excluded because of many artifacts related to "starting and finishing" the rest period.

\section{HRV analysis methods}

Frequency domain or power spectral density (PSD) analysis and time domain analysis are the two main analytical methods used to analyze
HRV [15]. In our study, we have focused on the power spectral analysis of HRV. Low frequency (LF, 0.04-0.15 Hz) and high frequency (HF, 0.15$0.4 \mathrm{~Hz}$ ), when expressed in their normalized units (n.u), are suggestive of the balanced behavior of sympathetic and parasympathetic branches of ANS. nLF is mainly contributed by sympathetic tone though some contribution from parasympathetic activity also exists. nHF is solely contributed by the parasympathetic tone and LFHF ratio gives an idea about the sympathovagal balance [16]

Subjects in the IAM group practiced the technique once daily and compliance was assessed by a self-maintained diary. Apart from assessing the diaries, compliance would also be assured by frequently telephoning the subjects and confirming that they are regularly practicing the technique. Furthermore, there were periodic refresher courses. Practicing the technique minimum 4 times a week was taken as the standard of compliance.

\section{Statistical analysis}

Statistical analysis was performed using IBM SPSS Version 20.0 software. Categorical variables are expressed using frequency and percentage. Continuous variables are presented using mean and standard deviation. To test the statistical significance of the mean changes of continuous variables between groups, the Mann-Whitney U-test was used. To test the statistical significance of the mean changes of continuous variables from the first visit to second visit within groups, the Wilcoxon signedrank test was used. $\mathrm{p}<0.05$ was considered as statistically significant.

\section{RESULTS}

Of the 30 type 2 diabetic subjects who were screened, informed, and assessed for eligibility for the study, 9 subjects did not meet the inclusion criteria, 2 gave reasons of travel and increased workload at the office, 1 had ill health, and 18 agreed to participate. However, on further being informed about the study intervention and time of the study, 8 immediately withdrew leaving 10 participants. They were randomized to two groups, namely, IAM group (test group) and control group with five in each arm. Subsequently, no further attrition occurred. Table 1 provides the sociodemographic characteristics of the study participants. The mean age of the IAM group subjects was found to be $50.40 \pm 8.41$ years and that of the control group subjects was $53.75 \pm 7.80$ years. $4: 1$ was the female-to-male ratio in the test group while $3: 2$ was their ratio in the control group. The groups were found to be comparable on the basis of age and sex ( $p>0.05$ ) (Table 1$)$.

In the diabetic test group, we could observe a statistically significant increase in the $\mathrm{HF}$ component ( $\mathrm{nHF}$ ) while a significant decrease in the LF component (nLF) after 6 months of study. Continuous IAM practice within the test group had significantly increased the parasympathetic tone as indicated by nHF from a baseline value of $21.72 \pm 9.47$ to $37.72 \pm 11.63(0.049)$ and nLF mainly suggestive of sympathetic tone had dropped from a baseline of $72.02 \pm 8.28$ to $60.50 \pm 11.98(0.036)$. The LF:HF ratio when compared within the test group also showed a significant decrease again suggesting the sympathovagal balance to more of parasympathetic activity. The LF:HF ratio had decreased from $3.85 \pm 1.62$ to $1.82 \pm 0.91$ over the study period $(0.024)$. Although not statistically significant, we could observe a decreasing trend for the nHF component and an increasing trend for $\mathrm{NLF}$ and LFHF ratio within the control group of diabetic subjects (Table 2).

Table 1: Sociodemographic characteristics of the study participants

\begin{tabular}{llll}
\hline Variables & $\begin{array}{l}\text { IAM group (n=5) } \\
\text { (mean } \pm \text { SD) }\end{array}$ & $\begin{array}{l}\text { Control } \\
\text { group (n=5) } \\
\text { (mean } \pm \text { SD) }\end{array}$ & p value \\
\hline Age (years) & $50.40 \pm 8.41$ & $53.75 \pm 7.80$ & 0.461 \\
Gender (Female:male) & $4: 1$ & $3: 2$ & 0.490 \\
\hline IAM: Integrated amrita meditation & &
\end{tabular}


When the mean percentage change of the frequency domain measures $\mathrm{nHF}, \mathrm{nLF}$, and LFHF ratio was analyzed between the two groups, we observed statistically significant differences for all three domains. Considering the nHF change, in the diabetic test group, an average percentage increase of $91.98 \pm 79.20$ was observed while in the control group, the mean percentage drop was $32.86 \pm 46.83$. This difference was statistically significant $(\mathrm{p}=0.021)$ between group comparison. Similarly, a significant difference was noted in the mean percentage change of nLF and LF HF measures between the groups. The average percentage drop of $n L F$ in the IAM group came to $16.16 \pm 11.79$ while the mean percentage increase of nLF in the control group was $11.07 \pm 17.44$. This change was again statistically significant between the groups $(\mathrm{p}=0.023)$. A similar change was noted in the mean percentage change for the LF:HF ratio also. The mean percentage drop in the LF-HF measure in the IAM group was $48.87 \pm 23.28$ while the control group showed an increase of $51.70 \pm 93.24 \%$, which was also statistically significant difference on between-group comparison ( $\mathrm{p}=0.047$ ) (Table 3 ).

\section{DISCUSSION}

Mind-body interventions are extremely beneficial in stress-related mental and physical disorders [17]. The question being addressed in this article is does IAM practice improve the autonomic balance toward parasympathetic dominance in Type 2 diabetic patients? Our pilot study was carried out to understand the effect of meditation practice on the overall autonomic balance of the individual and thereby substantiate the hypothesis put forward by eminent researchers in the field of complementary medicine and psychophysiology.

Although there is numerous literature that looked on the effect of various yoga and/or meditation techniques on HRV of normal individuals [2,18-20], its effect in the diabetic population is limited. Studies have proved that meditation programs are beneficial for diabetic patients in improving their glycemic control and increasing their adherence to treatment protocols by way of reducing their psychological stress levels $[21,22]$. The previous study on the impact of IAM on stress in type 2 diabetic subjects has shown that better glycemic control can be attained through the reduction of psychological stress by regular practice of IAM along with usual diabetic treatment regimens [23].

In our study, the increase in normalized HF in the meditation group after IAM technique was in line with the previous studies of meditation

Table 2: Comparison of frequency domain measures within each group

\begin{tabular}{|c|c|c|c|c|c|}
\hline \multirow[t]{2}{*}{ Variables } & \multirow[t]{2}{*}{ Visit } & $\begin{array}{l}\text { IAM group } \\
(n=5)\end{array}$ & \multirow[t]{2}{*}{ p value } & $\begin{array}{l}\text { Control } \\
\text { group }(n=5)\end{array}$ & \multirow[t]{2}{*}{$p$ value } \\
\hline & & $\begin{array}{l}\text { Mean } \pm \text { SD } \\
(\%)\end{array}$ & & $\begin{array}{l}\text { Mean } \pm \text { SD } \\
(\%)\end{array}$ & \\
\hline nLF & $\begin{array}{l}1 \\
2\end{array}$ & $\begin{array}{l}72.02 \pm 8.28 \\
60.50 \pm 11.98\end{array}$ & $0.036^{*}$ & $\begin{array}{l}46.12 \pm 25.50 \\
61.06 \pm 16.60\end{array}$ & 0.138 \\
\hline $\mathrm{nHF}$ & $\begin{array}{l}1 \\
2\end{array}$ & $\begin{array}{l}21.72 \pm 9.47 \\
37.72 \pm 11.63\end{array}$ & $0.049^{*}$ & $\begin{array}{l}52.42 \pm 25.95 \\
37.22 \pm 17.58\end{array}$ & 0.138 \\
\hline LFHF & $\begin{array}{l}1 \\
2\end{array}$ & $\begin{array}{l}3.85 \pm 1.62 \\
1.82 \pm 0.91\end{array}$ & $0.024^{*}$ & $\begin{array}{l}1.59 \pm 1.94 \\
2.11 \pm 1.27\end{array}$ & 0.345 \\
\hline
\end{tabular}

*Statistically significant. IAM: Integrated amrita meditation

Table 3: Comparison of mean percentage difference of frequency domain measures of HRV between two groups

\begin{tabular}{llll}
\hline Variable & $\begin{array}{l}\text { IAM group (n=5) } \\
\text { Mean } \pm \text { SD (\%) }\end{array}$ & $\begin{array}{l}\text { Control group (n=5) } \\
\text { Mean } \pm S D(\%)\end{array}$ & p value \\
\hline $\mathrm{nLF}$ & $-16.16 \pm 11.79$ & $11.07 \pm 17.44$ & $0.023^{*}$ \\
$\mathrm{nHF}$ & $91.98 \pm 79.20$ & $-32.86 \pm 46.83$ & $0.021^{*}$ \\
$\mathrm{LFHF}$ & $-48.87 \pm 23.28$ & $51.70 \pm 93.24$ & $0.047^{*}$ \\
\hline
\end{tabular}

*Statistically significant. IAM: Integrated amrita meditation, HRV: Heart rate variability and HRV. HF band is suggestive of the parasympathetic activity and is often called the respiratory band. Our study also showed a decrease in the LF band which represents more of a sympathetic tone along with some parasympathetic influence. The LF/HF ratio which primarily indicates the sympathovagal balance also showed a decrease in the meditation group after 6 months of intervention. Altogether these changes suggest the sympathovagal balance toward a parasympathetic activity.

Concordant findings were observed in a study that looked on the HRV of 36 subjects at baseline and after 10 days of intensive Vipassana meditation. The HF band increased as expected and the LF showed a decrease in the meditation task compared to resting baseline in their study. The normalized units of HRV were again concordant with other meditation studies. Zen meditation practice also showed a significant increase in the HF norms as well as a significant decrease in the LF norms [18].

In a study on heart rate variability by a mindfulness-based stress reduction program (MBSR), LF norm decreased and HF norm increased in 22 healthy volunteers following 8 weeks of intervention. This study also suggested that meditation could improve sympathovagal balance compared to controlled respiration alone [19]. Similar changes were observed following Qi gong and Kundalini meditation by Peng et al. when compared to controlled breathing $[24,25]$. A potent balance exists between sympathetic and parasympathetic nervous systems in a healthy, adaptable, and reactive nervous system. Any disharmony to this system will lead to a myriad of psychophysiological challenges to health and well-being [2].

The effect of two selected yogic breathing techniques on HRV was studied by Raghuraj et al. 1998. Kapalbadhi and Nadishudhi were the two breathing techniques used in their study. The results showed a significant increase in low-frequency (LF) power and LF/HF ratio while high-frequency (HF) power was significantly lower following Kapalabhati. An increased sympathetic activity accompanied by reduced vagal activity has been explained for this change in autonomic status during this particular breathing technique [20].

Polyvagal theory by Stephen Porges has been explained in the context of involvement of role of ANS particularly parasympathetic activity in behavioral changes, mood, and psychological flexibility. His polyvagal theory (1994) shows us that when faced with threat or danger, we first turn to our social engagement system to reestablish safety. If that does not bring us to safety, we turn to our fight/flight response. Finally, if that does not bring us to safety then our mind and body move into collapse and shut down stage [26]. If we could widen the social engagement system through meditation interventions that can promote the parasympathetic activity, it would be beneficial to the diabetic population in particular as they are exposed to repeated stress and trauma of lifelong medications which can end up in minimal resilience toward hardships in life.

\section{CONCLUSIONS}

Our study has shown demonstrable improvement in autonomic function which reflects reduced stress suggestive of enhanced parasympathetic activity after the practice of IAM in diabetic patients.

\section{CONFLICTS OF INTEREST}

The authors have declared that they have no conflicts of interest with respect to current research

\section{AUTHORS' CONTRIBUTIONS}

Contribution of Sarika K S includes literature search, data acquisition, manuscript preparation, and manuscript editing. Contributions of Vandana Balakrishnan, Harish Kumar, and Anand Kumar include the development of concepts, design, and manuscript review. Contribution of Sundaram K R includes statistical analysis of data. 


\section{AUTHORS' FUNDING}

This work was funded by seed grant support from Amrita Institute of Medical Sciences, Kochi, India.

\section{REFERENCES}

1. Hofmann SG, Sawyer AT, Witt AA, Oh D. The effect of mindfulnessbased therapy on anxiety and depression: A meta-analytic review. J Consult Clin Psychol 2010;78:169.

2. Krygier JR, Heathers JA, Shahrestani S, Abbott M, Gross JJ, Kemp AH. Mindfulness meditation, well-being, and heart rate variability: A preliminary investigation into the impact of intensive Vipassana meditation. Int J Psychophysiol 2013;89:305-13.

3. Geisler FC, Vennewald N, Kubiak T, Weber H. The impact of heart rate variability on subjective well-being is mediated by emotion regulation. Pers Individ Dif 2010;49:723-8.

4. Kashdan TB, Rottenberg J. Psychological flexibility as a fundamental aspect of health. Clin Psychol Rev 2010;30:865-78.

5. Lee ST, Hon EH. The fetal electrocardiogram: IV. Unusual variations in the QRS complex during labor. Am J Obstet Gynecol 1965;92:1140-8.

6. Ewing DJ, Burt AA, Williams IR, Campbell IW, Clarke BF. Peripheral motor nerve function in diabetic autonomic neuropathy. J Neurol Neurosurg Psychiatry 1976;39:453-60.

7. Schroeder EB, Chambless LE, Liao D, Prineas RJ, Evans GW, Rosamond WD, et al. Diabetes, glucose, insulin, and heart rate variability: The atherosclerosis risk in communities (ARIC) study. Diabetes Care 2005;28:668-74

8. Amos AF, McCarty DJ, Zimmet P. The rising global burden of diabetes and its complications: Estimates and projections to the year 2010. Diabet Med 1997;14:S7-85.

9. King H, Aubert RE, Herman WH. Global burden of diabetes, 19952025: Prevalence, numerical estimates, and projections. Diabet Care 1998;21:1414-31.

10. Zimmet P, Alberti KG, Shaw J. Global and societal implications of the diabetes epidemic. Nature 2001;414:782.

11. Gebel E. Diabetes Distress. Diabetes Forecast; 2013. Available from: http://www.diabetes.org/living-with-diabetes/complications/mentalhealth/diabetes-distress.html. [Last accessed on 2018 Jun 14].

12. Bali HK. Yoga-an ancient solution to a modern epidemic. Ready for prime time? Indian Heart J 2013;65:132.

13. Sripada R, Vangala HK, Padala S, Magharla DD. Non pharmacological approaches in the management of diabetes mellitus. Asian J Pharm Clin Res 2019;12:27-3.
14. Vandana B, Saraswathy L, Suseeladevi GK, Sundaram KR, Kumar H. Positive impact of integrated amrita meditation technique on heart rate, respiratory rate and IgA on young healthy adults. Tang Hum Med 2013;3:13-1

15. Vandana B, Vaidyanathan K, Saraswathy LA, Sundaram KR, Kumar H. Impact of integrated amrita meditation technique on adrenaline and cortisol levels in healthy volunteers. Evid Based Complement Alternat Med 2011;2011:1-6.

16. Malik M, Bigger JT, Camm AJ. Heart rate variability: Standards of measurement, physiological interpretation, and clinical use. Eur Heart J 1996; 17:354-81.

17. Brown RP, Gerbarg PL. Sudarshan Kriya yogic breathing in the treatment of stress, anxiety, and depression: Part I-neurophysiologic model. J Altern Complement Med 2005;11:189-201.

18. Wu SD, Lo PC. Inward-attention meditation increases parasympathetic activity: A study based on heart rate variability. J Biomed Res 2008;29:245-50.

19. Nijjar PS, Puppala VK, Dickinson O, Duval S, Duprez D, Kreitzer MJ, et al. Modulation of the autonomic nervous system assessed through heart rate variability by a mindfulness based stress reduction program. Int J Cardiol 2014;177:557-9.

20. Raghuraj P, Ramakrishnan AG, Nagendra HR, Telles S. Effect of two selected yogic breathing techniques on heart rate variability. Indian J Physiol Pharmacol 1998;42:467-72.

21. Varghese MP, Balakrishnan R, Pailoor S. Association between a guided meditation practice, sleep and psychological well-being in Type 2 diabetes mellitus patients. J Complement Integr Med 2018;15:0026.

22. Bairy S, Kumar AM, Raju MS, Achanta S, Naik B, Tripathy JP, et al. Is adjunctive naturopathy associated with improved glycaemic control and a reduction in need for medications among Type 2 diabetes patients? A prospective cohort study from India. BMC Complement Altern Med 2016;16:290.

23. Sarika KS, Balakrishnan V, Sundaram KR, Kumar H. Impact of integrated amrita meditation technique on stress in Type 2 diabetic patients. Indian J Med Res Forthcoming 2020.

24. Peng CK, Mietus JE, Liu Y, Khalsa G, Douglas PS, Benson H, et al. Exaggerated heart rate oscillations during two meditation techniques. Int J Cardiol 1999;70:101-7.

25. Peng CK, Henry IC, Mietus JE, Hausdorff JM, Khalsa G, Benson H, et al. Heart rate dynamics during three forms of meditation. Int $\mathrm{J}$ Cardiol 2004;95:19-27.

26. Porges SW. The polyvagal theory: Phylogenetic substrates of a social nervous system. Int J Psychophysiol 2001;42:123-46. 